\title{
Micro-grid Inverter Parallel Droop Control Method for Improving Dynamic Properties and the Effect of Power Sharing
}

\author{
Xiaohong Zhu \\ Qujing Power Supply Bureau, Qujing 655000, China
}

\begin{abstract}
Parallel operation control is one of the key techniques for high performance inverters with the features of excellent regulation, modular, high reliability, and redundancy. Through theoretical analysis of multi-inverters parallel operation model, the paper derived the conventional droop control method, the stability limit of this method and its small-signal model. the effect of different parameters on system power sharing is analyzed. The simulation of conventional droop control method with PSCAD software is also implemented. After discussing the limits of conventional droop control method, a novel adaptive droop control method, is proposed, which can improve active power dynamic and reactive power sharing. A small signal model is established and the advantages of the method over conventional droop control are also validated by simulation.
\end{abstract}

Keywords-inverter; droop control; wireless parallel; PSCAD simulation.

\section{INTRODUCTION}

With the gradual failure of conventional energy sources and the increase of environment pollution, countries around the world began to focus on eco-friendly, efficient and flexible power generations--Distributed Generation (DG). The rapid development of DG generations has provided a lot of clean and efficient energy for the community, but has also brought great challenges to the existing power system. In order to reduce the adverse impact on the existing distribution network bought by DG while give play to its auxiliary function, which is the effective use of renewable energy, micro-grid, as an important form of DG has gained attention and promotion from many countries around the world. Micro-grid is an individually controllable system composed of load and distributed power supply, and it provides electricity and heat to local load [1]. Micro-grid combines and connects various forms of energy and load into a power supply network by each controllable interface (usually power electronic interfaces). These power electronic interfaces are mostly inverters. In a micro-grid formed by multiple inverter interfaces, due to the differences in their output characteristics, they have a problem of circulation between inverters, which increase the burden on the current transformer and line loss, and it will affect the normal operation of the micro-grid in severe situations.

Meanwhile, with the rapid development of electrical equipment in modern society the demand for capacity of inverters is increasing. There are mainly two ways to improve the inverter capacity, one is designing high- power inverters and the other is to use inverter parallel technology to achieve power modular. Using standard inverter power modules in parallel can not only flexibly compose inverter power system of any desired capacity, while multiple parallel power supply modules share loads and main switches of each module is in the small current stress; thus the reliability is fundamentally ensured. And if necessary, we can parallel redundant inverters to obtain fault-tolerant redundant power in low cost in order to ensure the stability of critical power.

Based on the above understanding, the parallel inverter technology has become a hot research field of power electronics, which has great practical significance.

\section{PRINCIPLES AND LIMITATIONS OF CONVENTIONAL DROOP CONTROL PARALLEL SYSTEM}

\section{A. Parallel inverters' operation principles}

Parallel inverters working system is mainly composed of three parts (Figure 2-1): inverter modules, line impedances and load.

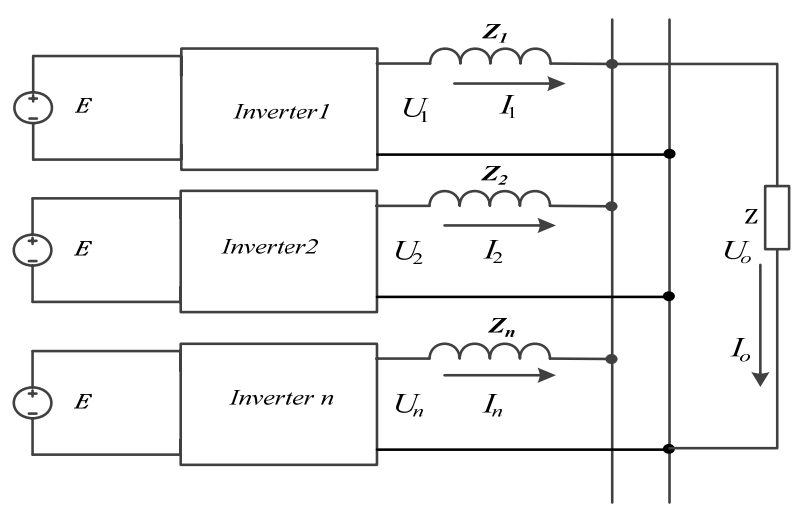

Figure 2-1. Parallel inverters' working system schematic

Parallel inverters that supply load, must be carried out with parallel control in order to meet certain conditions, which means that each inverter module's output voltage amplitude, frequency and phase need to be consistent to achieve current sharing control of each module. The main contents of this chapter focus on the model of the parallel inverters, and make analysis of power and circulation characteristics of the system, and then get the basic control strategy for parallel operation, and analyze power distribution and stability. 


\section{B. Control principles of droop control based parallel inverter system}

The following will be a detailed analysis of the theoretical principles of parallel inverter droop control.

The simplified schematic of parallel inverter system is shown in Figure 3-2.R1, X1 and R2, X2 are summations of each inverter's output connected impedance and line inductance.

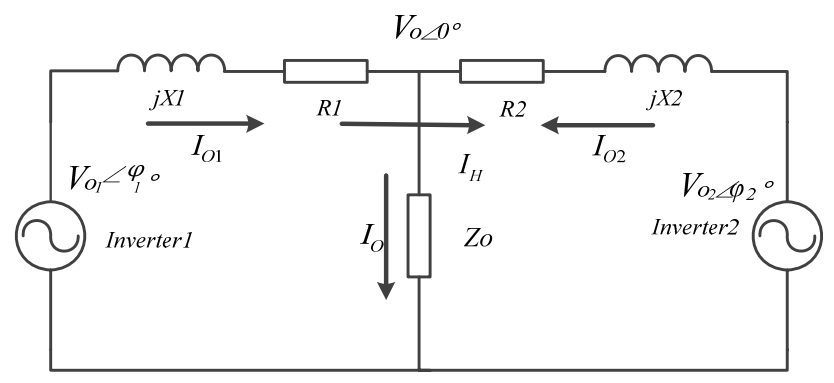

Figure 2-2. Two parallel inverter system diagram

$$
Z_{i} \angle \theta=R_{i}+j X_{i}(i=1,2)
$$

Complex power flowing into bus can be represented as:

$$
\mathrm{Si}=\mathrm{Pi}+\mathrm{jQi}
$$

Where:

Pi--Real power

Qi--Reactive power

Whose value is [7]

$$
\left\{\begin{array}{l}
P_{i}=\frac{V_{o i} V_{o}}{Z_{i}} \cos \left(\theta_{i}-\varphi_{i}\right)-\frac{V_{o}^{2}}{Z_{i}} \cos \theta_{i} \\
Q_{i}=\frac{V_{o i} V_{o}}{Z_{i}} \sin \left(\theta_{i}-\varphi_{i}\right)-\frac{V_{o}^{2}}{Z_{i}} \sin \theta_{i}
\end{array}\right.
$$

Where:

oi - ith inverter output voltage amplitude;

$\varphi \mathrm{i}$ - ith inverter power phase angle;

$\mathrm{Zi}$ - ith inverter output impedance value;

Voi - ith inverter output impedance phase;

Vo - common bus voltage

\section{Purely inductive output impedance case}

The traditional assumption considers the inverter output impedance is mainly inductive $\left(\theta=90^{\circ}\right)$, which is because that the inverter output filtering inductance is generally large, and also in the long-distance transmission, the line impedance is mainly inductive. In this case, we can derive the following expressions for active and reactive power from $(2-3)$ :

$$
\left\{\begin{array}{l}
P_{i}=\frac{V_{o i} V_{o}}{Z_{i}} \sin \varphi_{i} \\
Q_{i}=\frac{V_{o i} V_{o} \cos \varphi_{i}-V_{o}^{2}}{Z_{i}}
\end{array}\right.
$$

Generally, since $\varphi \mathrm{i}$ is small, $(\mathrm{i}=1,2)$ is relatively small, it can be approximated that: $\sin \varphi \approx 0, \cos \varphi \approx 1$, thus the active and reactive power is reduced to:

$$
\left\{\begin{array}{l}
P_{i}=\frac{V_{o i} V_{o}}{X_{i}} \varphi_{i} \\
Q_{i}=\frac{V_{o}\left(V_{o i}-V_{o}\right)}{X_{i}}
\end{array}\right.
$$

For equation (2-5), the real power output of the inverter is a function of its output voltage amplitude and power angle, differential on both sides, we get:

$$
\Delta p_{o i}=\frac{V_{o}}{X_{i}}\left(V_{o i} * \Delta \varphi_{i}+\varphi_{i}^{*} \Delta V_{o i}+\Delta V_{o i}^{*} * \Delta \varphi_{i}\right)
$$

Since $\varphi$ i is very small, Simplify Eq.(2-5) we get:

$$
\Delta p_{o i}=\frac{V_{o} V_{o i}}{X_{i}} * \Delta \varphi_{i}
$$

Similarly, differential reactive power on both sides, we get:

$$
\Delta q_{o i}=\frac{V_{o}}{X_{i}} * \Delta V_{o i}
$$

From Eq.(2-7)\&(2-8) we know that the output voltage phase affects its output real power, while the output voltage amplitude change its output reactive power. Thus, to control the inverter output real power and reactive power, this can simply be achieved by adjusting the amplitude and phase of inverter output voltage. However, due to the phase is not easy to detect, it is generally by adjusting the frequency of the output voltage to regulate the the phase of the output voltage, and thus to regulate the real power output of the inverter.

Frequency and amplitude of the output voltage of each inverter varies as the following equation, where we get the conventional droop control equation:

$$
\begin{gathered}
\omega_{i}=\omega_{o i}-m_{i} * P_{i} \\
V_{i}=V_{o i}-n_{i}^{*} Q_{i}
\end{gathered}
$$

Where:

Mi -droop factor of output angular frequency of the ith inverter (referred as frequency droop factor)

$\mathrm{Ni}$ - droop factor of output voltage amplitude of the ith inverter (referred as voltage droop factor)

\section{Purely resistive output impedance case}

In the low-voltage distribution lines, the impedance of the line is mainly resistive $\left(\theta=0^{\circ}\right)$. Same as the derivation in 2.2.1, we can get: 


$$
\left\{\begin{array}{l}
P_{i}=\frac{V_{o}\left(V_{o i}-V_{o}\right)}{R_{i}} \\
Q_{i}=-\frac{V_{o i} V_{o}}{R_{i}} \varphi_{i}
\end{array}\right.
$$

Thus, when the output impedance is highly resistive, $\mathrm{P}$ / Q droop objects need to be changed:

$$
\left\{\begin{array}{l}
\omega_{i}=\omega_{o i}+m_{i} * Q_{i} \\
V_{i}=V_{o i}-n_{i} * P_{i}
\end{array}\right.
$$

Therefore, P- $\omega$ and Q-V droop based control strategy should be applied to inductive output impedance, and for resistive output impedance, $\mathrm{P}-\mathrm{V}$ and $\mathrm{Q}-\mathrm{W}$ droop based control should be applied.

\section{E. The power distribution relationship of conventional droop control based inverter}

1) Distribution of real power

As shown in Figure 2-2, two parallel inverter system, when steady state is reached, the frequencies of two inverters are inevitable equal, otherwise the system will be in a dynamic adjustment or oscillation process. So there is:

$$
\omega_{1}=\omega_{2}
$$

Substituting Eq.(2-18) in the the Eq.(2-9), we can get the expression of real power distribution of two inverters in steady-state:

$$
\omega_{o 1}-m_{1} * P_{1}=\omega_{o 2}-m_{2} * P_{2}
$$

It shows that real power of two steady-state inverters is only related to its frequency and frequency droop factor of no-load output voltage, regardless of the output inductance.

2) Reactive power distribution

When two inverters reached steady state, the inverter output voltage amplitude is shown in Eq.(2-3). Substitute Eq.(2-10) into Eq.(2-3), we have:

$$
Q_{i}=\frac{V_{o i} \cos \varphi_{i}-V_{o}}{\frac{X_{i}}{V_{o}}+n_{i} \cos \varphi_{i}}
$$

Clearly, in addition to controllable parameters Voi and ni, reactive power is also affected by output inductance. Thus, when the two output impedances of inverters are imbalance, reactive power cannot be well shared.

\section{THE PROPOSED IMPROVED DROOP CONTROL METHOD}

\section{A. Limitations of virtual impedance control}

The implementation of control method of last chapter is too complicate. Differentiator must be used if virtual impedance is added, and differentiators easily enlarge high-frequency noise, which affect the system's stability. Moreover, these controls have high requirements on controllers' speed, which increase the cost of the controllers. This chapter proposed two easily implemented improvements of control methods. The figure below is a schematic diagram of the proposed improved control strategy.

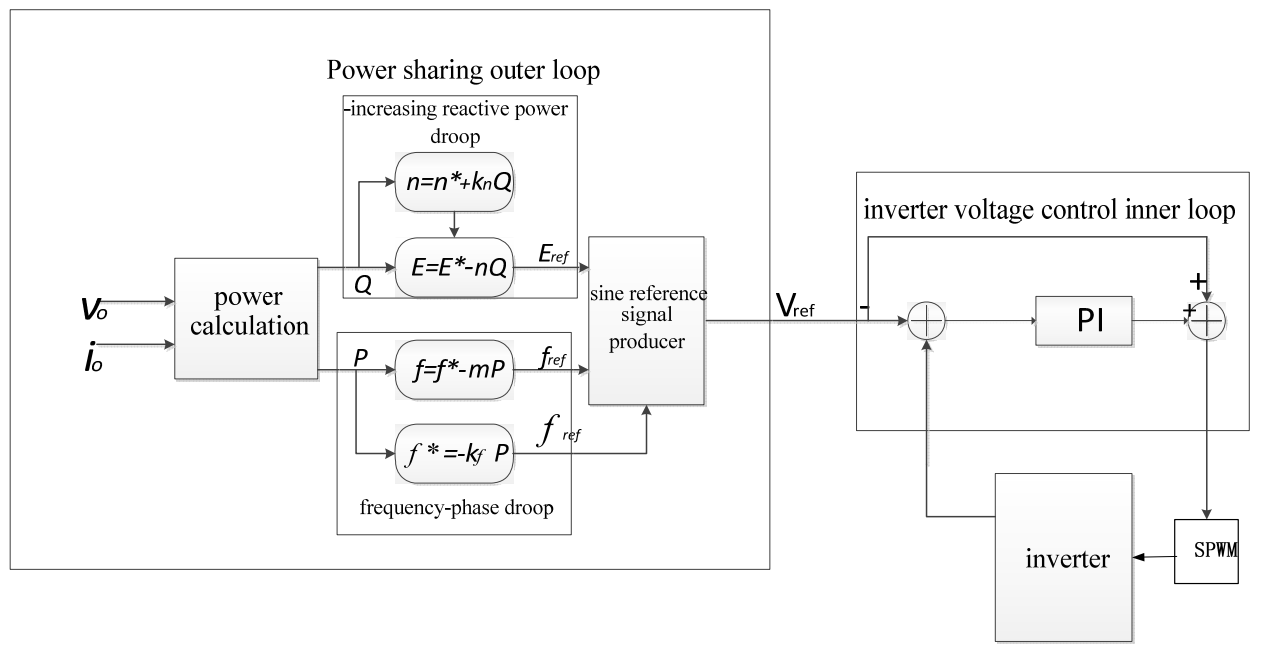

Figure 3.1. Block diagram of the proposed improved closed-loop droop control system

B. Control strategy to improve the dynamic characteristics of real power

1) Analysis of initial phase droop method

The phase of the inverter output voltage is $\varphi$, using conventional droop control, as the equation:

$$
\varphi=\int \omega d t=\int\left(\omega^{*}-m P\right) d t=m \int\left(\frac{\omega^{*}}{m}-P\right) d t
$$

In conventional droop control, the phase control is pure integral control, the dynamic following capability of sudden power change of pure integral control is not as 
good as the dynamic following capability of proportional integral control. If the following real power droop strategy is applied:

$$
\left\{\begin{array}{l}
\omega=\omega^{*}-m P \\
\varphi^{*}=-k_{\varphi} P
\end{array}\right.
$$

Where $\varphi^{*}$ is the generated initial phase of the reference voltage. Then

$$
\varphi=\int \omega d t+\varphi^{*}=\int\left(\omega^{*}-m P\right) d t-k_{\varphi} P-=m \int\left(\frac{\omega^{*}}{m}-P\right) d t-k_{\varphi} P
$$

After adding the initial phase droop, the control form of the phase is a proportional integral control.

$$
\omega=\frac{d \varphi}{d t}=\omega^{*}-m P-\frac{d \varphi^{*}}{d t}=\omega^{*}-m P-k_{\varphi} \frac{d P}{d t}
$$

For frequency $\omega$, it means the introduction of real power derivative term. This control method which directly adds the derivative term is also proposed in some literatures. However, with respect to the direct introduction of real power differential term, the proposed initial phase droop method of this work can use a simple linear expression to achieve the same effect without adding a differentiator, which also avoids the highfrequency noise caused by the differential terms.

2) Simulation of initial phase droop method

Main circuits of initial phase droop method's simulation are as follows:

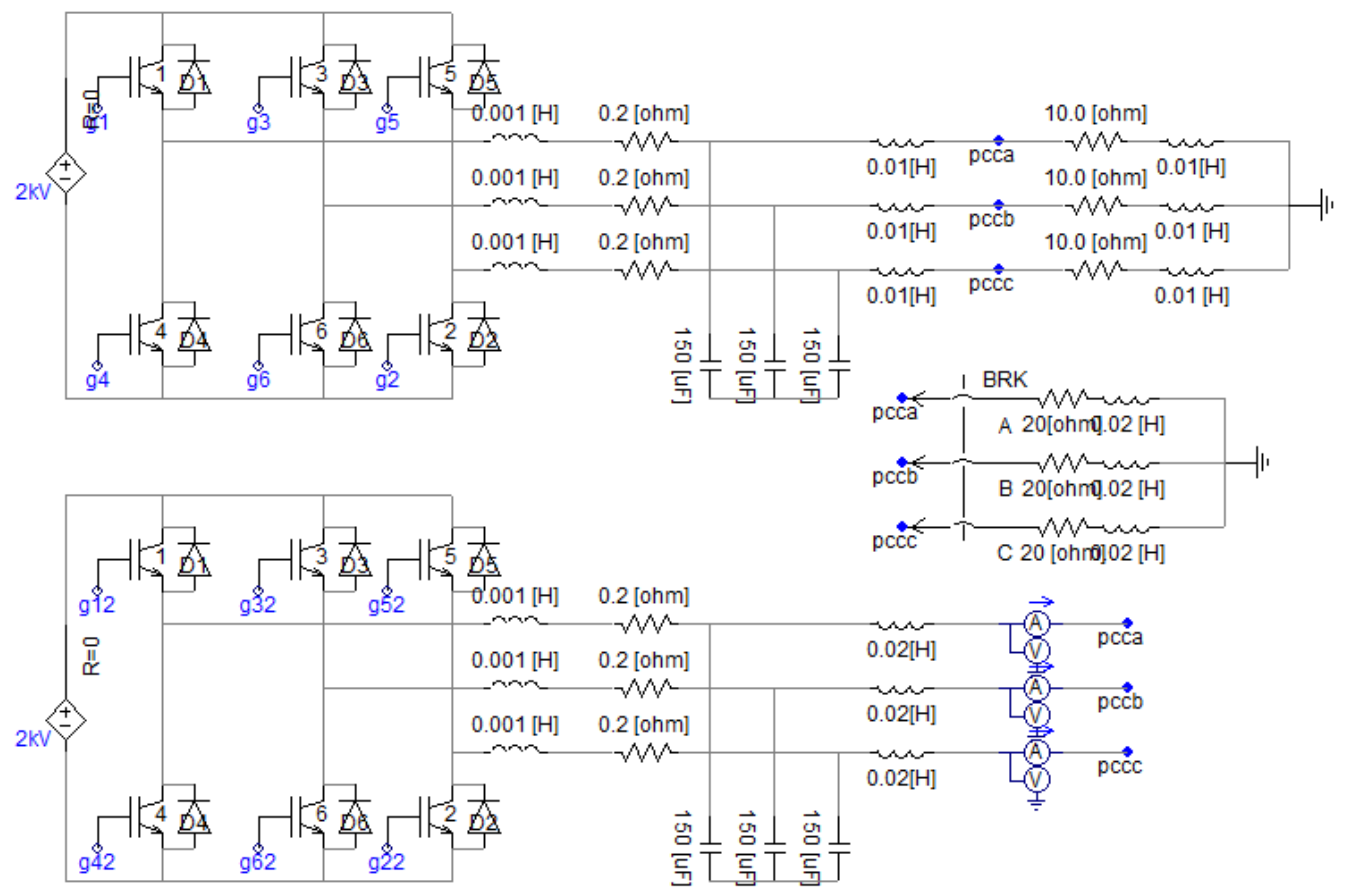

Figure 3-2. Main simulation circuit (line impedances of two inverters are different, $0.5 \mathrm{~S}$ breaker is closed, sudden load increase)

The simulation parameters are shown in Table3-1.

TABLE 3-1. SIMULATION PARAMETERS

\begin{tabular}{llllllll}
\hline & $f^{*}(\mathrm{~Hz})$ & $m$ & $E^{*}(k)$ & $n$ & $k_{i}$ & $k_{p}$ & $k_{\varphi}$ \\
\hline Inv1 & 50.35 & 10.0 & 0.818 & 1 & 20 & 0.4 & 100 \\
Inv 2 & 50.35 & 10.0 & 0.818 & 1 & 20 & 0.4 & 100 \\
\hline
\end{tabular}

As can be seen from the simulation results in Figure 33 , the real power dynamic characteristics is greatly improved after adding initial phase droop control, and the adjusting time is significantly reduced. 


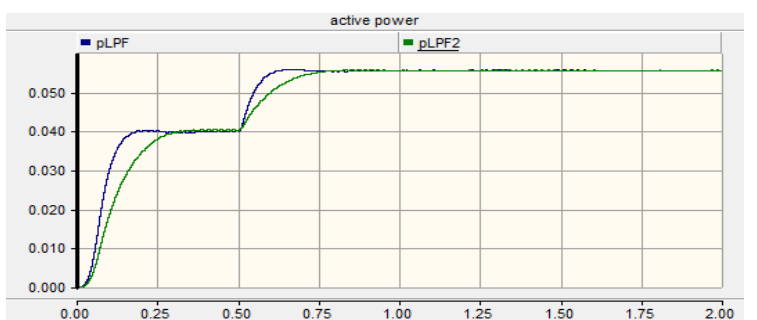

(a)

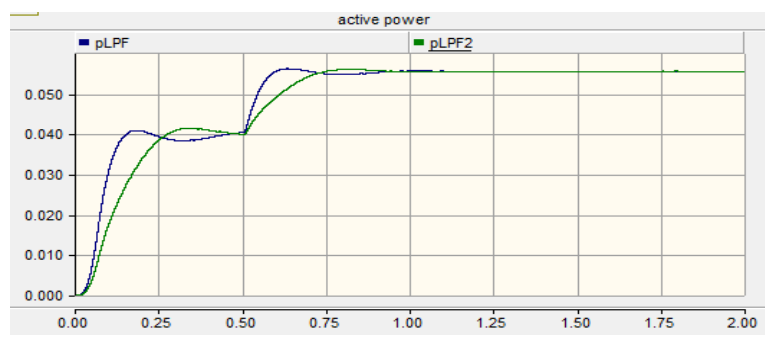

(b)

\section{Control strategy of improving reactive power sharing effect}

Obtained by the analysis in Section 3.4, when the two inverter output impedances are unequal, even by using droop control cannot share reactive power well. In inductive output impedances, reactive power can be controlled either by controlling the output voltage or the output impedance. In addition, the effect of reactive power sharing can be enhanced by control the voltage droop factor. If the droop factor is not a constant, but increases with the reactive power, as shown in equation.

$$
n_{i}=n_{i}^{*}+k_{n} Q_{i}
$$

Figure 3-3. Simulation of real power curve of initial phase droop (a. Conventional droop b. Initial phase droop)

TABLE 3-2. SimUlation PARAMETERS

\begin{tabular}{llllllllll}
\hline & $f^{*}(\mathrm{~Hz})$ & $m$ & $E^{*}(\mathrm{kV})$ & $n$ & $k_{i}$ & $k_{p}$ & $k_{\varphi}$ & $k_{n}$ & $n^{*}$ \\
\hline $\operatorname{Inv} 1$ & 50.3745 & 10.0 & 0.8148 & 1 & 20 & 0.4 & 100 & 500 & 1 \\
$\operatorname{Inv} 2$ & 50.3745 & 10.0 & 0.8148 & 1 & 20 & 0.4 & 100 & 500 & 1 \\
\hline
\end{tabular}

Then simulation below is to used to verify the effect of improving reactive power control. The simulation parameters are shown in Table 3-2. With this control, two inverters' voltage droop factor are no longer equal, as shown in Figure 3-4. Reactive power line graphs of before and after adding droop are shown in Figure 3-5. Clearly, the effect of reactive power sharing has been greatly improved.

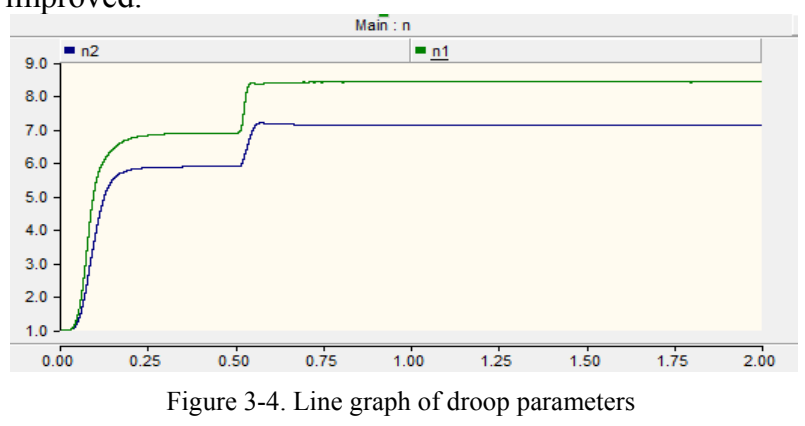

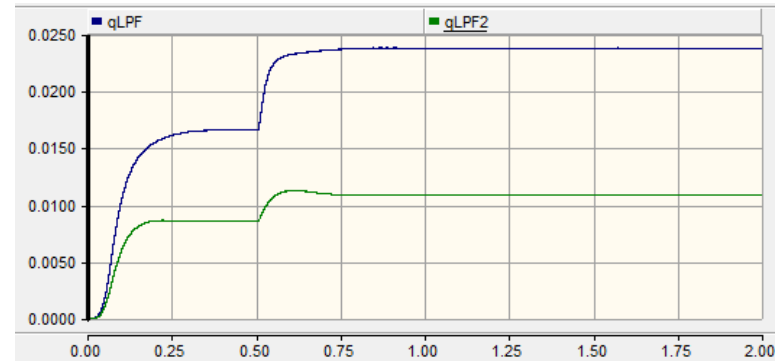

(a)

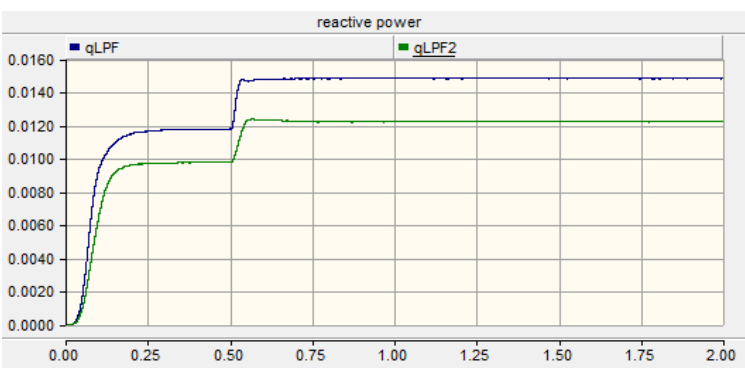

(b)

Figure 3-5:. Simulation reactive power line graph of increased droop factors

(a. Conventional droop b. Increased droop factors y-axis:. MVA; $\mathrm{x}$-axis: s) 


\section{CONCLUSION}

This paper analyzed the impact on droop control and inverter sharing that caused by different situations of transmission lines, and made improvement for droop control when transmission line inductance values are not equal. Improvements for the former one is power of the outer ring, which introduces reactive power droop control system to increase the effect of improvement of reactive power sharing. And for the slow flow of power rings and shock easily, initial phase droop control is introduced, which improved real power dynamic characteristic. Both control strategies are verified and simulated by PSCAD.

\section{REFERENCES}

[1] R.Lasseter, A.Abbas, C.Marnay, and et al. Integration of distributed energy resources. California Energy Commission, 2003.
[2] J.M. Guerrero, L.G. de Vicuna, J. Matas, et al. Output Impedance Design of Parallel-Connected UPS Inverters With Wireless LoadSharing Control. IEEE Trans. on Industrial Electronics, 2005, 52(4): 1126-1135.

[3] Chiang S J, Chang J M. Parallel control of the UPS inverters with frequency-dependent droop scheme. IEEE Annual Power Electronics Specialists Conference, Vancouver, Canada, 2001

[4] De Brabandere K, Bolsens B, Van den Keybus J, et al. A voltage and frequency droop control method for parallel inverters. IEEE Annual Power Electronics Specialists Conference, Aachen, Germany, 2004.

[5] J.M. Guerrero, L.G. de Vicuna, J. Matas, et al. A Wireless Controller to Enhance Dynamic Performance of Parallel Inverters in Distributed Generation Systems. IEEE Trans. on Power IEEE Trans. on Power Electronicsvol, 2004, 19(5): 1205-1213. 